\title{
STABILIZATION OF A HYBRID SYSTEM WITH A NONLINEAR NONMONOTONE FEEDBACK
}

\author{
EDUARD FEIREISL ${ }^{1}$ AND GEOFFREY O'DOWD ${ }^{2}$
}

\begin{abstract}
For a hybrid system composed of a cable with masses at both ends, we prove the existence of solutions for a class of nonlinear and nonmonotone feedback laws by means of a priori estimates. Assuming some local monotonicity, strong stabilization is obtained thanks to some Riemann's invariants technique and La Salle's principle.

Résumé. Pour un système hybride constitué d'un câble aux extrémités duquel sont accrochées deux masses, on prouve l'existence de solutions pour une classe de lois de feedbacks non linéaires et non monotones grâce à des estimations a priori. Une hypothèse de monotonie locale combinée à une technique d'invariants de Riemann permet de se ramener à un semi-groupe contractant pour lequel le principe de La Salle conduit à la stabilité forte du système.
\end{abstract}

AMS Subject Classification. 93D15, 47H20.

Received March 27, 1998. Revised November 30, 1998.

\section{INTRODUCTION}

In this work, we study an overhead crane model consisting of a cable carrying a load, the cable being linked at its top end to a platform moving along a rail by means of a feedback type force taking into account the position and the velocity of the platform. The objective is to drive it at a given point from a given configuration so that the whole structure should be at rest.

In Section 2, we describe a modelization and mention some previous results, while in Section 3, we convert this system in terms of an evolution equation for which well-posedness is proven using a result of lipschitz perturbation of maximal monotone operators and some a priori estimates . The essential part of this work is Section 4, which is devoted to establishing the strong stabilization of the hybrid system.

\section{DescRiption OF THE MODEL}

We consider the modelization described in [1,6] or [4]: $m$ and $M$ denote respectively the mass of the platform and the load. The cable is non stretching and of unit length.

Let us define $y(x, t)$ as the horizontal displacement at the point of the cable whose curvilinear abscissa is $x$ at time $t$ and $a(x)$ the tension force of the cable. The force acting on the platform is noted $F$.

Keywords and phrases: Hybrid systems, wave equation, stabilization by feedback law, Riemann invariants

${ }^{1}$ Institut of Mathematics AVCR, Žitna 25, 11567 Prague 1, République Tchèque; e-mail: feireisl@math.cas.cz

2 Institut Élie Cartan, BP. 239, 54506 Vandœuvre-lès-Nancy, France; e-mail: odowd@iecn.u-nancy.fr

(c) EDP Sciences, SMAI 1999 
Assuming that the oscillations of the cable are weak, this system is governed by the equations

$$
\left\{\begin{array}{l}
y_{t t}(x, t)-\left(a y_{x}\right)_{x}(x, t)=0, t>0,0<x<1 \\
\left(a y_{x}\right)(1, t)+M y_{t t}(1, t)=0 \\
\left(a y_{x}\right)(0, t)-m y_{t t}(0, t)=F(t) .
\end{array}\right.
$$

The wave equation is coupled with dynamical equations at both ends creating thus a hybrid system.

We shall suppose throughout this work that the tension force satisfies

$$
\begin{aligned}
& \text { (i) } a \in H^{1}(0,1) \\
& \text { (ii) } a(x) \geq a_{0}>0 \forall x \in[0,1] .
\end{aligned}
$$

For a solution $y$ of (2.1), let us define its energy:

$$
E(t)=\frac{1}{2}\left[\int_{0}^{1}\left(a(x) y_{x}^{2}(x, t)+y_{t}^{2}(x, t)\right) d x+\alpha y^{2}(0, t)+m y_{t}^{2}(0, t)+M y_{t}^{2}(1, t)\right]
$$

where $\alpha>0$ is a constant. As we seek for asymptotic stabilization at $y=0$, we shall prove that under suitable assumptions $E(t)$ tends to zero as $t$ goes to infinity.

A simple formal computation gives

$$
E^{\prime}(t)=-y_{t}(0, t)(F(t)-\alpha y(0, t))
$$

so that choosing $F$ of the form

$$
F(t)=\alpha y(0, t)+f\left(y_{t}(0, t)\right)
$$

where the feedback function $f$ satisfies

$$
\forall s \in \mathbb{R}, s f(s) \geq 0
$$

we obtain dissipativity of the system, since

$$
E^{\prime}(t)=-y_{t}(0, t) f\left(\left(y_{t}(0, t)\right) \leq 0 .\right.
$$

In [1], by neglecting $M$ and taking $f$ non decreasing, the authors prove the well-posedness and strong stabilization of the system. The same results are obtained in [6] by neglecting $m$; moreover, estimates of the energy decay are obtained according to the behaviour of $f$ at 0 and infinity.

In [5], both masses are taken into account and strong stabilization is achieved when $f$ is linear but no decay rate may be expected according to Russell's compact perturbation of a semigroup of isometric operators theorem (see [7]).

In these works, strong stabilization is essentially due to La Salle's principle (see [3]) applied to a semigroup which is contractant by virtue of the monotonicity assumption on the feedback law $f$. We shall prove that the global monotonicity assumption may be relaxed to a local one.

\section{WELL-POSEDNESS OF THE SYSTEM}

Let us from now on study the system

$$
\left\{\begin{array}{l}
y_{t t}(x, t)-\left(a y_{x}\right)_{x}(x, t)=0 \\
\left(a y_{x}\right)(1, t)+M y_{t t}(1, t)=0 \\
\left(a y_{x}\right)(0, t)-m y_{t t}(0, t)=\alpha y(0, t)+f\left(y_{t}(0, t)\right)
\end{array}\right.
$$


which is converted in a standard fashion into an evolution equation by introducing the variable $U(t)=$ $\left(y(., t), y_{t}(., t), y_{t}(0, t), y_{t}(1, t)\right)$. More precisely, let us define the space

$$
H=H^{1}(0,1) \times L^{2}(0,1) \times \mathbb{R}^{2}
$$

endowed with the hilbertian scalar product:

$$
\langle U, \tilde{U}\rangle=\int_{0}^{1}\left[a(x) y_{x}(x) \tilde{y}_{x}(x)+z(x) \tilde{z}(x)\right] d x+\alpha y(0) \tilde{y}(0)+m \eta \tilde{\eta}+M \xi \tilde{\xi}
$$

for $U=(y, z, \eta, \xi)$ and $\tilde{U}=(\tilde{y}, \tilde{z}, \tilde{\eta}, \tilde{\xi})$, the unbounded operator $A_{0}$

$$
\begin{gathered}
D\left(A_{0}\right)=\left\{U=(y, z, \eta, \xi) \in H^{2}(0,1) \times H^{1}(0,1) \times \mathbb{R}^{2} / \eta=z(0), \xi=z(1)\right\} \\
A_{0}(U)=\left(-z,-\left(a y_{x}\right)_{x},-\frac{1}{m}\left(a y_{x}(0)-\alpha y(0)\right), \frac{1}{M} a y_{x}(1)\right)
\end{gathered}
$$

and the operator $B$ defined on $H$ by

$$
B(U)=\left(0,0, \frac{1}{m} f(\eta), 0\right)
$$

We consider now the evolution equation

$$
\frac{d U}{d t}+A_{0} U+B(U)=0, U(0)=U_{0}
$$

with initial data $U_{0}=\left(y_{0}, z_{0}, \eta_{0}, \xi_{0}\right) \in D\left(A_{0}\right)$.

As one can easily check, a solution $t \mapsto U(t)=(y(., t), z(., t), \eta(t), \xi(t))$ of (3.7) is such that $y$ satisfies (3.1), at least formally, with $y(x, 0)=y_{0}(x)$ and $y_{t}(x, 0)=z_{0}(x)$. For this reason, system (3.1) will be interpreted in terms of equation (3.7).

Next, we prove the existence and uniqueness of solutions for (3.7) by means of a lipschitz perturbation theorem under the following assumptions for the feedback law $f$ :

(i) $\quad f \in C^{0}(\mathbb{R})$ and $\forall s \in \mathbb{R}, s f(s) \geq 0$

(ii) on every compact interval, the increment ratio $\frac{f(s)-f\left(s^{\prime}\right)}{s-s^{\prime}}$ is bounded below.

Lemma 1. The operator $A_{0}$ defined by (3.4)-3(5) is maximal monotone on $H$.

Proof. Monotonicity: a straightforward computation gives $\left\langle A_{0} U, U\right\rangle=0$ for all $U_{0} \in D\left(A_{0}\right)$. The monotonicity of $A_{0}$ is then a consequence of its linearity.

Maximality: let $U_{0}=\left(y_{0}, z_{0}, \eta_{0}, \xi_{0}\right) \in H$. The equation

$$
\left(I+A_{0}\right) U=U_{0}, U \in D\left(A_{0}\right)
$$


is equivalent to finding $y \in H^{2}(0,1)$ and $z \in H^{1}(0,1)$ such that

$$
\left\{\begin{array}{cc}
y-z & =y_{0} \\
z-\left(a y_{x}\right)_{x} & =z_{0} \\
z(0)-\frac{1}{m}\left(a y_{x}(0)-\alpha y(0)\right) & =\eta_{0} \\
z(1)+\frac{1}{M} a y_{x}(1) & =\xi_{0} .
\end{array}\right.
$$

Eliminating $z=y-y_{0}$, one obtains

$$
\left\{\begin{array}{cl}
y-\left(a y_{x}\right)_{x} & =y_{0}+z_{0} \\
\frac{1}{m} a y_{x}(0)-\left(\frac{\alpha}{m}+1\right) y(0) & =-\eta_{0}-y_{0}(0) \\
\frac{1}{M} a y_{x}(1)+y(1) & =\xi_{0}+y_{0}(1)
\end{array}\right.
$$

By applying Lax-Milgram's theorem, (3.11) has a unique solution $y \in H^{2}(0,1)$. Setting $z=y-y_{0}, \eta=z(0)$ and $\xi=z(1)$, we see that $U=(y, z, \eta, \xi)$ satisfies $(3.9)$.

For the existence of solutions for equation (3.7), we need the following result (see for instance [2], Th. 3.17 p. 105 and Prop. 3.2 p. 67):

Theorem 1. Let $H$ be a Hilbert space, $A$ a maximal monotone operator on $H$ with dense domain $D(A)$ and $L$ a lipschitz operator defined on $H$. Then for all $U_{0} \in D(A)$, there exists a unique function $U:[0,+\infty[\rightarrow H$ satisfying:

$$
\begin{array}{ll}
\text { (i) } & U(0)=U_{0} \\
\text { (ii) } & U(t) \in D(A) \text { for all } t \geq 0 \\
\text { (iii) } & \forall T>0, U \in W^{1, \infty}((0, T), H) \\
\text { (iv) } & \frac{d U}{d t}(t)+A U(t)+L U(t)=0 \text { for a.e. } t \in[0,+\infty[.
\end{array}
$$

Now we are ready to prove:

Theorem 2. Assume (3.8). Then for all $U_{0} \in D\left(A_{0}\right)$, equation (3.7) admits a unique strong solution $t \mapsto U(t)$ satisfying

$$
\forall t \geq 0, U(t) \in D\left(A_{0}\right) \text { and } \forall T>0, U \in W^{1, \infty}((0, T), H) .
$$

Set, for all $t \geq 0$ and for all $U_{0} \in D\left(A_{0}\right), S(t)\left(U_{0}\right)=U(t)$. Then $(S(t))_{t \geq 0}$ can be extended to a (possibly non contractive) semigroup of strongly continuous operators on $H$.

Proof. The essential argument relies on an a priori estimate, which enables us to write $A_{0}$ as the sum of a maximal monotone operator and a lipschitz operator and then use Theorem 1.

Given any $U_{0} \in D\left(A_{0}\right)$, a solution $U=(y, z, \eta, \xi)$ of (3.7) satisfies

$$
\left\langle\frac{d U}{d t}, U\right\rangle=\left\langle-A_{0} U, U\right\rangle-\langle B(U), U\rangle=-\frac{1}{m} f(\eta) \eta \leq 0
$$

so that $\frac{1}{2}\|U(t)\|^{2}$ is non increasing. Thus,

$$
\|U(t)\|^{2} \leq\|U(0)\|^{2}=\left\|U_{0}\right\|^{2} .
$$

In particular, by $(3.3),|\eta(t)| \leq \frac{1}{\sqrt{m}}\left\|U_{0}\right\|=K$. This shows that the values of $f$ out of $I_{K}=[-K, K]$ have no influence on equation (3.7). In other terms, let us give any $\bar{f}$ which coincides with $f$ on $I_{K}$ and set $\bar{B}$ the 
operator defined on $H$ by $\bar{B}(U)=\left(0,0, \frac{1}{m} \bar{f}(\eta), 0\right)$. Then any solution $\bar{U}$ of

$$
\frac{d \bar{U}}{d t}+A_{0} \bar{U}+\bar{B}(\bar{U})=0, \bar{U}(0)=U_{0}
$$

is a solution of (3.7) and vice versa.

Now, according to (3.8)(ii), there exists $c_{K} \geq 0$ such that $\frac{f(s)-f\left(s^{\prime}\right)}{s-s^{\prime}} \geq-c_{K}$ on $I_{K}$. Thus, the function $f_{1}$ : $s \mapsto f(s)+c_{K} s$ is non decreasing on $I_{K}$. Let $\bar{f}_{1}$ be any continuous non decreasing extension of $f_{1}$ to $\mathbb{R}$ and define $\bar{f}: s \mapsto \bar{f}_{1}(s)-c_{K} s$. As previously said, the resolution of (3.7) is equivalent to the resolution of (3.13) with $\bar{B}$ associated to that $\bar{f}$.

Next, write $\bar{B}=B_{1}+L$, where $B_{1}(U)=\left(0,0, \frac{1}{m} f_{1}(\eta), 0\right)$ and $L(U)=\left(0,0,-\frac{1}{m} c_{K} \eta, 0\right)$. Operator $L$ is clearly lipschitz on $H$ and it is easy to check that $B_{1}$, with domain $H$, is a maximal monotone operator on $H$. It then follows from [4], Corollary 2.7, p. 36, that $A=A_{0}+B_{1}$ with domain $D\left(A_{0}\right)$ is maximal monotone on $H$. Thus, the existence, uniqueness and regularity of a solution for equation (3.13), and consequently (3.7), follows from Theorem 1.

Next, $A_{0}$ being a linear maximal monotone operator, its domain $D\left(A_{0}\right)$ is dense in $H$. Finally, let us give $R>0$. According to the a priori estimate discussed above and adopting the same notations, there exist a maximal monotone operator $A$ with domain $D\left(A_{0}\right)$ and a lipschitz operator $L$, both depending on $R$, such that for every $\left(U_{0}, V_{0}\right) \in D\left(A_{0}\right)^{2}$ whose $H$-norm are less than $R$, the solutions $U(t), V(t)$ of (3.7) with respective initial conditions $U_{0}, V_{0}$ coincide with the solutions $\bar{U}(t), \bar{V}(t)$ of (3.13) with the same resp. initial conditions.

We then have

$$
\begin{aligned}
\left\langle\frac{d U}{d t}-\frac{d V}{d t}, U-V\right\rangle & =\left\langle\frac{d \bar{U}}{d t}-\frac{d \bar{V}}{d t}, \bar{U}-\bar{V}\right\rangle \\
& =-\langle A \bar{U}-A \bar{V}, \bar{U}-\bar{V}\rangle-\langle L(\bar{U})-L(\bar{V}), \bar{U}-\bar{V}\rangle \leq c\|\bar{U}-\bar{V}\|^{2}=c\|U-V\|^{2},
\end{aligned}
$$

where $c$ is the lipschitz constant of $L$; this shows that the function

$$
t \mapsto e^{-2 c t}\|U(t)-V(t)\|^{2}
$$

is non increasing. Hence, $\|U(t)-V(t)\| \leq e^{c t}\left\|U_{0}-V_{0}\right\|$ i.e.

$$
\left\|S(t)\left(U_{0}\right)-S(t)\left(V_{0}\right)\right\| \leq e^{c t}\left\|U_{0}-V_{0}\right\| .
$$

This lipschitz property of the operators $S(t)$ on every $H$-bounded subset of $D\left(A_{0}\right)$ leads easily to the extension of $S(t)$ into a bounded operator on $H$, with the semigroup property.

To end this section, let us precise the exact connexion between system (3.1) and equation (3.7):

Proposition 1. For all $U_{0}=\left(y_{0}, z_{0}, \eta_{0}, \xi_{0}\right) \in D\left(A_{0}\right)$, the solution $t \mapsto U(t)=(y(., t), z(., t), \eta(t), \xi(t))$ of (3.7) is such that:

(i) $\forall T>0, y \in W^{2, \infty}\left((0, T), L^{2}(0,1)\right) \cap W^{1, \infty}\left((0, T), H^{1}(0,1)\right)$,

(ii) $\quad \forall T>0, \eta, \xi \in W^{1, \infty}((0, T), \mathbb{R}), \forall t \geq 0, y(, . t) \in H^{2}(0,1)$,

(iii) for a.e. $(x, t) \in(0,1) \times \mathbb{R}^{+}, y_{t t}(x, t)-\left(a(x) y_{x}(x, t)\right)_{x}=0$,

(iv) $\quad$ for a.e. $t \in \mathbb{R}^{+}, \eta(t)=y_{t}(0, t), \xi(t)=y_{t}(1, t)$

(v) $\quad a(0) y_{x}(0, t)-m \eta^{\prime}(t)=\alpha y(0, t)+f\left(y_{t}(0, t)\right)$

(vi) $\quad a(1) y_{x}(1, t)+M \xi^{\prime}(t)=0$,

(vii) $y(., 0)=y_{0}, y_{t}(., 0)=z_{0}$.

Moreover, the function $E\left[U_{0}\right]: t \mapsto \frac{1}{2}\|U(t)\|^{2}$ has a derivative on $\mathbb{R}^{+}$and

$$
E\left[U_{0}\right]^{\prime}(t)=y_{t}(0, t) f\left(y_{t}(0, t)\right) \leq 0 .
$$


Proof. It's a direct interpretation of operators $A_{0}$ and $B$. Regularity is provided by Theorem 2. However, without more regularity for $y$, no sense can a priori be given neither to $y_{t t}(0, t)$ nor too $y_{t}(1, t)$. Lastly, $(3.14)$ follows from the regularity of $y$ and an integration by parts as already observed in (3.12).

\section{Strong stabilization}

A standard method in that kind of dissipative system is to use La Salle's invariance principle, as in [6]. This requires usually the contraction property of the semigroup associated to the system, which is lacking in our case. As it can be easily shown, the non decreasingness of the feedback law $f$ would imply the contraction of the semigroup, since $c$ can be taken as zero in the proof of Theorem 2. In fact, we may have contraction in a certain sense with only local monotonicity for $f$ around zero.

Let us consider these properties:

(4.1) there exists $\delta>0$ such that $f$ is strictly increasing on $]-\delta, \delta[$,

(4.2) there exists $\delta>0$ such that $f$ is strictly increasing on $] 0, \delta[$,

and $f(s)=0$ for all $s \leq 0$,

(4.3) there exists $\delta>0$ and $D>0$ such that $f$ is strictly increasing on

] $0, \delta[$ and on $]-D-\delta,-D[$ and $f(s)=0$ for all $s \in[-D, 0]$.

The intervals $]-\delta, \delta[$ in (4.1), ] $-\infty, \delta[$ in (4.2) and $]-D-\delta, \delta[$ in (4.3) will be called domain of monotonicity of $f$.

The main result of the paper is the following:

Theorem 3. Assume (3.8) and either (4.1, 4.2, 4.3). Then for all $U_{0} \in D\left(A_{0}\right)$, the solution $t \mapsto U(t)$ of (3.7) satisfies:

$$
\|U(t)\| \rightarrow 0 \text { as } t \rightarrow+\infty .
$$

Let us sketch the outlines of the proof: the essential idea is to show that for large enough $t, S(t)$ acts on $U_{0}$ as a contractive semigroup. This will be a consequence of the decisive Lemma 4 . Proof of Theorem 3 will then follow from Theorem 4 concerning stabilization of a contractive semigroup by means of La Salle's invariance principle.

Lemma 4 is mainly due to the estimations of Lemma 3, which are the consequence of the introduction of the Riemann invariants of Lemma 2. Let us now pass to the details.

Lemma 2. Let $y$, with the regularity of Proposition 1 (i) and (ii), be a solution on $(0,1) \times \mathbb{R}^{+}$of the wave equation

$$
y_{t t}-\left(c^{2} y_{x}\right)_{x}=0
$$

with $c \in H^{1}(0,1)$ and $c(x) \geq c_{0}>0$. Let $\varphi$ be a primitive of $-\frac{1}{c}, K$ a constant which is greater than $\max _{x \in[0,1]}|\varphi(x)-\varphi(0)|$ and define the functions $g$ and $h$ by:

$$
\begin{aligned}
& g(x, t)=y_{t}(x, t+\varphi(x)-\varphi(0)+K)+c(x) y_{x}(x, t+\varphi(x)-\varphi(0)+K), \\
& h(x, t)=y_{t}(x, t-\varphi(x)+\varphi(0)+K)-c(x) y_{x}(x, t-\varphi(x)+\varphi(0)+K) .
\end{aligned}
$$

Then for a.e. $(x, t) \in(0,1) \times \mathbb{R}^{+}$:

$$
\begin{aligned}
& g_{x}(x, t)=-c^{\prime}(x) y_{x}(x, t+\varphi(x)-\varphi(0)+K) \\
& h_{x}(x, t)=c^{\prime}(x) y_{x}(x, t-\varphi(x)+\varphi(0)+K) .
\end{aligned}
$$

The proof is immediate. It should be noticed that in case $c$ is constant, one recovers the classical Riemann's invariants, namely: $y_{t}(x, t) \pm c y_{x}(x, t)$ is constant along the characteristics $x \pm c t=$ constant. In fact, only $g$ will be later used.

From now on, we fix $U_{0} \in D\left(A_{0}\right)$ and use notations of Proposition 1. 
Lemma 3. There exist constants $\beta$ and $\gamma>0$, depending quadratically on $\left\|U_{0}\right\|$ on the coefficient $a$ and on the function $f$ as well as a constant $K>0$ depending on a such that for any bounded interval $I \subset \mathbb{R}^{+}$,

$$
\int_{I} \eta^{\prime}(t+K) d t \leq \beta|I|+\gamma
$$

Proof. Obviously, we have already from the dissipativity (3.14): $\left|y^{2}(0, t)\right| \leq \frac{1}{\alpha}\left\|U_{0}\right\|^{2},\left|y_{t}^{2}(0, t)\right| \leq \frac{1}{m}\left\|U_{0}\right\|^{2}$, and by the continuity of $f,\left|f^{2}\left(y_{t}(0, t)\right)\right| \leq M_{0}=\max |f|^{2}$ on the interval $\left[-\frac{1}{\sqrt{m}}\left\|U_{0}\right\|, \frac{1}{\sqrt{m}}\left\|U_{0}\right\|\right]$. Hence the following inequalities hold

$$
\int_{I} y^{2}(0, t) d t, \int_{I} y_{t}^{2}(0, t) d t, \int_{I} f^{2}\left(y_{t}(0, t)\right) d t \leq \beta|I|+\gamma
$$

for suitable constants $\beta$ and $\gamma$. So according to Proposition 1(v), it is sufficient to prove that the estimate

$$
\int_{I} y_{x}^{2}(0, t) d t \leq \beta|I|+\gamma
$$

holds. For that purpose, we shall make use of Lemma 2 where we set $c=\sqrt{a}$ which, according to (2.2) and [6] Corollary VIII.10 p. 131 , belongs to $H^{1}(0,1)$ and satisfies $c(x) \geq c_{0}>0$.

We use the notations of Lemma 2. Writing $g(x, t)-g(x, 0)=\int_{0}^{x} g_{x}(s, t) d s$ and applying Cauchy Schwarz's inequality, one obtains

$$
g^{2}(0, t) \leq 2\left(g^{2}(x, t)+\left[\int_{0}^{1}\left|g_{x}(s, t)\right| d s\right]^{2}\right) .
$$

Consequently, for any interval $I=[A, A+T] \subset \mathbb{R}^{+}$we have:

$$
\begin{aligned}
\int_{A}^{A+T} g^{2}(0, t) d t= & \int_{0}^{1} \int_{A}^{A+T} g^{2}(0, t) d t d x \leq 2\left[\int_{0}^{1} \int_{A}^{A+T} g^{2}(x, t) d t d x+\int_{A}^{A+T}\left(\int_{0}^{1}\left|g_{x}(s, t)\right| d s\right)^{2} d t\right] \\
\leq & 4 \int_{0}^{1} \int_{A}^{A+T}\left[y_{t}^{2}(x, t+\varphi(x)-\varphi(0)+K)+c^{2}(x) y_{x}^{2}(x, t+\varphi(x)-\varphi(0)+K)\right] d t d x \\
& +2 \int_{A}^{A+T}\left(\int_{0}^{1}\left|c^{\prime}(s) y_{x}(s, t+\varphi(s)-\varphi(0)+K)\right| d s\right)^{2} d t
\end{aligned}
$$

Let us note $4 I_{1}+2 J_{1}$ the right hand side of (4.7). Setting $x=s$ and applying Cauchy-Schwarz's inequality, we have

$$
\begin{aligned}
J_{1} & \leq \int_{0}^{1}{c^{\prime}}^{2}(x) d x \int_{A}^{A+T} \int_{0}^{1} y_{x}^{2}(x, t+\varphi(x)-\varphi(0)+K) d x d t \\
& =\left\|c^{\prime}\right\|_{L^{2}}^{2} \int_{0}^{1} \int_{A}^{A+T} y_{x}^{2}(x, t+\varphi(x)-\varphi(0)+K) d t d x .
\end{aligned}
$$

With $x$ being fixed, the change of variable $v=t+\varphi(x)-\varphi(0)+K$ (with respect to $t$ ) leads to

$$
\int_{A}^{A+T} y_{x}^{2}(x, t+\varphi(x)-\varphi(0)+K) d t=\int_{A+\varphi(x)-\varphi(0)+K}^{A+T+\varphi(x)-\varphi(0)+K} y_{x}^{2}(x, v) d v .
$$


Thanks to the choice of $K, \varphi(x)-\varphi(0)+K \geq 0$; moreover, there exists a constant $C_{2}$, depending only on the function $\varphi$ such that $\varphi(x)-\varphi(0)+K \leq C_{2}$. In other words,

$$
[A+\varphi(x)-\varphi(0)+K, A+T+\varphi(x)-\varphi(0)+K] \subset\left[A, A+T+C_{2}\right]
$$

and $y_{x}^{2}$ being $\geq 0,(4.8)$ gives

$$
\int_{A}^{A+T} y_{x}^{2}(x, t+\varphi(x)-\varphi(0)+K) d t \leq \int_{A}^{A+T+C_{2}} y_{x}^{2}(x, v) d v .
$$

Thus,

$$
J_{1} \leq\left\|c^{\prime}\right\|_{L^{2}}^{2} \int_{0}^{1} \int_{A}^{A+T+C_{2}} y_{x}^{2}(x, v) d v .
$$

Proceeding in the same fashion for $I_{1}$, we get:

$$
I_{1} \leq \int_{0}^{1} \int_{A}^{A+T+C_{2}}\left[y_{t}^{2}(x, v)+c^{2}(x) y_{x}^{2}(x, v)\right] d v d x .
$$

Inserting estimates (4.9) and (4.10) into (4.7) yields

$$
\begin{aligned}
\int_{A}^{A+T} g^{2}(0, t) d t \leq & 4\left(\int_{0}^{1} \int_{A}^{A+T+C_{2}}\left[y_{t}^{2}(x, v)+c^{2}(x) y_{x}^{2}(x, v)\right] d v d x\right. \\
& \left.+\left\|c^{\prime}\right\|_{L^{2}}^{2} \int_{0}^{1} \int_{A}^{A+T+C_{2}} y_{x}^{2}(x, v) d x d v\right) .
\end{aligned}
$$

Using the fact that $c(x) \geq c_{0}>0$ as well as Fubini's theorem, we deduce from (4.11) the estimate

$$
\int_{A}^{A+T} g^{2}(0, t) d t \leq C\left(\int_{A}^{A+T+C_{2}} \int_{0}^{1}\left[y_{t}^{2}(x, v)+c^{2}(x) y_{x}^{2}(x, v)\right] d x d v\right),
$$

the constant $C$ depending only on the function $c=\sqrt{a}$.

Finally, according to the definition of $\|\cdot\|$ and using (3.12), we get

$$
\int_{A}^{A+T} g^{2}(0, t) d t \leq C \int_{A}^{A+T+C_{2}}\left\|U_{0}\right\|^{2} d v=C\left(T+C_{2}\right)\left\|U_{0}\right\|^{2} .
$$

As $g(0, t)=y_{t}(0, t+K)+c(0) y_{x}(0, t+K)$, we obtain (4.6) using the second estimate of (4.5) and proof of Lemma 3 is complete.

Lemma 4. There exists a time $t_{0}>0$, depending only on $U_{0}$, such that for all $t \geq t_{0}, y_{t}(0, t)$ belongs to the domain of monotonicity of $f$.

Proof. Fix any $d>\frac{2}{\delta}\left(\frac{1}{\alpha}\left\|U_{0}\right\|\right)^{\frac{1}{2}}$, where $\delta$ is defined in (4.1-4.3), consider any sequence $\left(a_{n}\right)$ of limit $+\infty$ and define the sequence of functions $\left(v_{n}\right)$ on $[0, d]$ by

$$
v_{n}(t)=\eta\left(t+a_{n}+K\right)=y_{t}\left(0, t+a_{n}+K\right) .
$$

According to Proposition 1 (ii), $v_{n} \in H^{1}(0, d)$ and thanks to $(4.4),\left(v_{n}\right)$ is a bounded sequence in $H^{1}(0, d)$. Due to the compactness of the injection $H^{1}(0, d) \hookrightarrow C([0, d])$, there exists a subsequence, still noted $\left(v_{n}\right)$, converging uniformly to a continuous function $v$ on $[0, d]$. 
Next, we prove that $f(v(t))$ is identically 0 on $[0, d]$. For that purpose, we observe that $v$ cannot satisfy $\min |v| \geq \delta$, otherwise

$$
\begin{aligned}
d \delta & >2\left(\frac{1}{\alpha}\left\|U_{0}\right\|\right)^{\frac{1}{2}} \geq\left|y\left(0, a_{n}+d+K\right)-y\left(0, a_{n}+K\right)\right| \\
& =\left|\int_{a_{n}+K}^{a_{n}+K+d} y_{t}(0, t) d t\right|=\left|\int_{0}^{d} v_{n}(t) d t\right| \underset{n \rightarrow \infty}{\longrightarrow}\left|\int_{0}^{d} v(t) d t\right| \geq d \delta,
\end{aligned}
$$

which is a contradiction. Thus

$$
L=v([0, d]) \cap]-\delta, \delta[\text { is a nonvoid interval. }
$$

On the other hand, (3.14) gives, since $s f(s) \geq 0$,

$$
\int_{0}^{+\infty} y_{t}(0, t) f\left(y_{t}(0, t)\right) d t \leq E(0)<+\infty
$$

which implies

$$
\lim _{n \rightarrow+\infty} \int_{a_{n}}^{a_{n}+K+d} y_{t}(0, t) f\left(y_{t}(0, t)\right) d t=0
$$

Hence,

$$
\begin{aligned}
\int_{0}^{d} v(t) f(v(t)) d t & =\lim _{n \rightarrow+\infty} \int_{0}^{d} v_{n}(t) f\left(v_{n}(t)\right) d t \\
& =\lim _{n \rightarrow+\infty} \int_{a_{n}}^{a_{n}+K+d} y_{t}(0, t) f\left(y_{t}(0, t)\right) d t=0 .
\end{aligned}
$$

Since $t \mapsto v(t) f(v(t))$ is continuous and $\geq 0$, we deduce that for all $t \in[0, d]$, $v(t) f(v(t))=0$ and as $f(0)=0$,

$$
\forall t \in[0, d], f(v(t))=0 .
$$

For the rest of the proof of Lemma 4, we distinguish three cases.

- If $f$ satisfies (4.1), then (4.14) together with (4.16) gives $L=\{0\}$, therefore $v([0, d])=\{0\}$ i.e. $v \equiv 0$. The sequence $\left(a_{n}\right)$ being arbitrary, we deduce easily

$$
y_{t}(0, t) \underset{t \rightarrow \infty}{\longrightarrow} 0 .
$$

Otherwise, there would exist $\varepsilon>0$ and a sequence $t_{n} \rightarrow+\infty$ such that $\left|y_{t}\left(0, t_{n}+K\right)\right| \geq \varepsilon$. The sequence $v_{n}: t \mapsto y_{t}\left(0, t_{n}+t+K\right)$ on $[0, d]$ would converge (after extraction of a subsequence) to zero uniformly on $[0, d]$. In particular, $v_{n}(0) \rightarrow 0$ as $n \rightarrow+\infty$; but $\left|v_{n}(0)\right|=\left|y_{t}\left(0, t_{n}+K\right)\right| \geq \varepsilon$, which is a contradiction.

Lemma 4 is then proven in that case, since $\left|y_{t}(0, t)\right|<\delta$ for large enough $t$.

- If $f$ satisfies (4.3), we deduce from (4.14) and (4.16) that:

$$
v([0, d]) \cap[0, \delta[=\{0\} \quad \text { and } \quad v([0, d]) \cap]-D-\delta, 0[\subset[-D, 0[.
$$

Obviously, this implies that $v([0, d]) \subset[-D, 0]$. Using the same argument as in the previous case, there can't exist $\varepsilon>0$ and arbitrary large $t$ such that $y_{t}(0, t+K) \geq \varepsilon$ or $y_{t}(0, t+K) \leq-D-\varepsilon$. Thus, for large enough $t, y_{t}(0, t+K) \in[-D-\delta, \delta]$. 
- If $f$ satisfies (4.2), the proof is quite the same.

Proof of Lemma 4 is now complete.

At this stage, we are able to recover the general setup of a contractive semigroup as follows: consider any non decreasing continuous function $\bar{f}$ on $\mathbb{R}$ which coincides with $f$ on its domain of monotonicity. In particular,

$$
\forall s>0, \bar{f}(s)>0
$$

Define, as in the proof of Theorem 2, the operator $\bar{B}: U \mapsto\left(0,0, \frac{1}{m} \bar{f}(\eta), 0\right)$ on $H$, which is maximal monotone; the operator $A_{0}+\bar{B}$, with domain $D\left(A_{0}\right)$, being maximal monotone on $H$, the equation of evolution

$$
\frac{d \bar{U}}{d t}+\left(A_{0}+\bar{B}\right) \bar{U}=0, \bar{U}(0)=\bar{U}_{0}
$$

possesses a unique solution for all $U_{0} \in D\left(A_{0}\right)$ with the regularity

$$
\begin{aligned}
& \text { (i) } \quad \forall t \geq 0, \bar{U}(t) \in D\left(A_{0}\right) \\
& \text { (ii) } \bar{U} \in W^{1, \infty}((0,+\infty), H) \cap L^{\infty}\left((0,+\infty), D\left(A_{0}\right)\right)
\end{aligned}
$$

given by $\bar{U}(t)=\bar{S}(t) \bar{U}_{0}$, where $(\bar{S}(t))_{t>0}$ is the contractive semigroup associated to operator $A_{0}+\bar{B}$.

Considering the solution of (4.18) with the initial condition $\bar{U}(0)=U\left(t_{0}\right)$, state of the solution of (3.7) at instant $t_{0}$ for which $y_{t}(0, t)$ 'enters' in the domain of monotonicity of $f$, we see that for all $t \geq 0, \bar{U}(t)=U\left(t+t_{0}\right)$, thanks to the definition of $\bar{f}$ and the uniqueness of the Cauchy problem associated to an evolution equation governed by a maximal monotone operator.

Thus, Theorem 3 will be a consequence of

Theorem 4. For all $\bar{U}_{0} \in D\left(A_{0}\right)$, the solution $\bar{U}$ of (4.18) satisfies

$$
\|\bar{U}(t)\| \underset{t \rightarrow \infty}{\longrightarrow} 0
$$

Proof of Theorem 4 is somewhat classical: we first establish in Lemma 5 that the $\omega$-limit sets are non empty and consider the solutions of (4.18) of constant energy in Lemmas 6 and 7 . We then conclude applying La Salle's principle.

Lemma 5. (i) The canonical embedding from $D\left(A_{0}\right)$, equipped with the graph norm, into $H$ is compact.

(ii) For all $\bar{U}_{0} \in D\left(A_{0}\right)$, the set $\omega\left(\bar{U}_{0}\right)=\left\{\bar{W} \in H / \exists\left(t_{n}\right) \rightarrow+\infty\right.$ such that $\left.\bar{U}\left(t_{n}\right) \rightarrow \bar{W}\right\}$ is non empty, included in $D\left(A_{0}\right)$, invariant under the action of $(\bar{S}(t))_{t \geq 0}$ and satisfies $\operatorname{dist}\left(\bar{S}(t)\left(\bar{U}_{0}\right), \omega\left(\bar{U}_{0}\right)\right) \rightarrow 0$ as $t \rightarrow+\infty$.

(iii) For all $\bar{U}_{0} \in D\left(A_{0}\right)$ and for all $\bar{W} \in \omega\left(\bar{U}_{0}\right), t \mapsto\|\bar{S}(t) \bar{W}\|^{2}$ is constant.

Proof. We see easily from the definition of operator $A_{0}$ that the graph topology is equivalent to the topology endowed by the injection of $D\left(A_{0}\right)$ in $H^{2}(0,1) \times H^{1}(0,1) \times \mathbb{R}^{2}$, hence (i).

(ii) follows from classical dynamical systems theory and (iii) from La Salle's principle applied to the Lyapounov functional $t \mapsto E(t)$.

In the following lemma, we determine the solutions of (4.18) of constant energy in the case corresponding to assumption (4.1).

Lemma 6. Let $\bar{U}(t)=(\bar{y}(., t), \bar{z}(., t), \bar{\eta}(t), \bar{\xi}(t))$ be a solution of (4.18) with the regularity (4.19) such that

$$
\forall t \geq 0, \bar{y}_{t}(0, t)=0 \text {. }
$$

Then $\bar{y}$ is identically zero. 
The proof is based on a multiplier method. For the details, we refer to [6] or [5].

Next, we consider the solutions of (4.18) of constant energy in the cases corresponding to assumptions (4.2) or (4.3).

Lemma 7. Let $\bar{U}(t)=(\bar{y}(., t), \bar{z}(., t), \bar{\eta}(t), \bar{\xi}(t))$ be a solution of (4.18) with the regularity (4.19) such that

$$
\forall t \geq 0, \bar{y}_{t}(0, t) \leq 0 \text { and } \bar{f}\left(\bar{y}_{t}(0, t)\right)=0 \text {. }
$$

Then $\bar{y}_{t}(0, t) \rightarrow 0$ as $t \rightarrow+\infty$.

Proof. As for equation (3.7), we have $\|\bar{U}(t)\| \leq\|\bar{U}(0)\|$ and then $|\bar{y}(0, t)| \leq \frac{1}{\sqrt{\alpha}}\|\bar{U}(0)\|$. Since $\bar{y}_{t}(0, t) \leq 0$, we deduce that $\bar{y}(0, t)$ is nonincreasing and bounded on $\mathbb{R}^{+}$so that

$$
l=\lim _{t \rightarrow+\infty} \bar{y}(0, t)
$$

exists. Moreover, since $\bar{y}_{t t}-\left(a \bar{y}_{x}\right)_{x}=0$, we have, for all $T>0$,

$$
\begin{aligned}
0 & =\int_{0}^{1} \int_{0}^{T}\left(\bar{y}_{t t}-\left(a \bar{y}_{x}\right)_{x}\right) d t d x=\int_{0}^{1}\left[\bar{y}_{t}(x, t)\right]_{0}^{T} d x-\int_{0}^{T}\left[a \bar{y}_{x}(x, t)\right]_{0}^{1} d t \\
& =\int_{0}^{1}\left(\bar{y}_{t}(x, T)-\bar{y}_{t}(x, 0)\right) d x-\int_{0}^{T} a \bar{y}_{x}(1, t) d t+\int_{0}^{T} a \bar{y}_{x}(0, t) d t \\
& =\int_{0}^{1}\left(\bar{y}_{t}(x, T)-\bar{y}_{t}(x, 0)\right) d x+M \int_{0}^{T} \bar{\xi}^{\prime}(t) d t+\int_{0}^{T}\left(m \bar{\eta}^{\prime}(t)+\alpha \bar{y}(0, t)\right) d t \\
& =\int_{0}^{1}\left(\bar{y}_{t}(x, T)-\bar{y}_{t}(x, 0)\right) d x+M \bar{y}_{t}(1, t)-M \bar{y}_{t}(1,0)+m \bar{y}_{t}(0, T)-m \bar{y}_{t}(0,0)+\int_{0}^{T} \alpha \bar{y}(0, t) d t .
\end{aligned}
$$

Thus,

$$
\int_{0}^{T} \alpha \bar{y}(0, t) d t=-\left[\int_{0}^{1}\left[\bar{y}_{t}(x, T)-\bar{y}_{t}(x, 0)\right] d x+M \bar{y}_{t}(1, T)-M \bar{y}_{t}(1,0)+m \bar{y}_{t}(0, T)-m \bar{y}_{t}(0,0)\right]
$$

From (4.19), we deduce easily that $\int_{0}^{1}\left[\bar{y}_{t}(x, T)-\bar{y}_{t}(x, 0)\right] d x$ is bounded independently of $T$, as well as $M \bar{y}_{t}(1, T)-$ $M \bar{y}_{t}(1,0)+m \bar{y}_{t}(0, T)-m \bar{y}_{t}(0,0)$. We then deduce from (4.20) that

$$
\left|\int_{0}^{T} \bar{y}(0, t) d t\right| \text { is bounded independently of } T
$$

which is only compatible with $l=0$. And since $\bar{y}(0, t)$ is non increasing, we deduce that $\bar{y}(0, t) \geq 0$ and

$$
\int_{0}^{+\infty} \bar{y}(0, t) d t<+\infty
$$

Integrating by parts gives

$$
\int_{0}^{T} \bar{y}_{t}^{2}(0, t) d t=\left[\bar{y}(0, t) \bar{y}_{t}(0, t)\right]_{0}^{T}-\int_{0}^{T} \bar{y}(0, t) \bar{\eta}^{\prime}(t) d t
$$


and from (4.19) we have $\bar{\eta} \in W^{1, \infty}\left(\mathbb{R}^{+}, \mathbb{R}\right)$, so $\left|\bar{\eta}^{\prime}\right| \leq C_{2}$ and we deduce from (4.23) that

$$
\int_{0}^{T} \bar{y}_{t}^{2}(0, t) d t \leq C_{0} C_{1}+C_{2} \int_{0}^{+\infty} \bar{y}(0, t) d t
$$

this shows that

$$
\int_{0}^{+\infty} \bar{y}_{t}^{2}(0, t) d t<+\infty
$$

As in the proof of Lemma 4 , consider any sequence $a_{n} \rightarrow+\infty$ and the sequence of functions $\left(\bar{v}_{n}\right)$ defined on $[0,1]$ by $\bar{v}_{n}(t)=\bar{\eta}\left(t+a_{n}\right)=\bar{y}_{t}\left(0, t+a_{n}\right)$. Since $\bar{\eta}$ and $\bar{\eta}^{\prime}$ are bounded on $\mathbb{R}^{+}$,

$$
\begin{aligned}
& \int_{0}^{1} \bar{v}_{n}^{2}(0, t) d t=\int_{a_{n}}^{a_{n}+1} \bar{\eta}^{2}(t) d t \leq C_{3}, \\
& \int_{0}^{1}{\overline{v^{\prime}}}_{n}^{2}(0, t) d t=\int_{a_{n}}^{a_{n}+1}{\overline{\eta^{\prime}}}^{2}(t) d t \leq C_{4} .
\end{aligned}
$$

Thus, $\left(\bar{v}_{n}\right)$ is a bounded sequence in $H^{1}(0,1)$ and there exists a subsequence, still noted $\left(\bar{v}_{n}\right)$, which converges uniformly to a continuous function $\bar{v}$ on $[0,1]$. Hence,

$$
\int_{0}^{1} \bar{v}^{2}(t) d t=\lim _{n \rightarrow+\infty} \int_{0}^{1} \bar{v}_{n}^{2}(t) d t=\lim _{n \rightarrow+\infty} \int_{a_{n}}^{a_{n}+1} \bar{y}_{t}^{2}(0, t) d t
$$

and thanks to (4.25), this last limit is zero, so we deduce that $\bar{v}$ is identically zero on $[0,1]$ which means that $\bar{y}_{t}\left(0, t+a_{n}\right)$ vanishes uniformly on $[0,1]$. The sequence $\left(a_{n}\right)$ being arbitrary, we deduce that

$$
\bar{y}_{t}(0, t) \underset{t \rightarrow \infty}{\longrightarrow} 0 .
$$

Let us finally return Theorem 4 which will be entirely proved, according to Lemma 5(ii), when we show that $\omega\left(\bar{U}_{0}\right)=\{0\}$ for all $\bar{U}_{0} \in D\left(A_{0}\right)$.

Let $\bar{U}_{1} \in \omega\left(\bar{U}_{0}\right)$ and define $\bar{V}(t)=\bar{S}(t) \bar{U}_{1}=(\bar{y}(., t), \bar{z}(., t), \bar{\eta}(t), \bar{\xi}(t))$ the solution of (4.18) with initial condition $\bar{U}_{1}$. According to Lemma $5($ iii) $), t \mapsto\|\bar{V}(t)\|^{2}$ is constant; its derivative $-2 \bar{y}_{t}(0, t) \bar{f}\left(\bar{y}_{t}(0, t)\right)$ is therefore zero which means according to (4.17) that

$$
\forall t \geq 0, \bar{y}_{t}(0, t) \leq 0 \text { and } \bar{f}\left(\bar{y}_{t}(0, t)\right)=0 .
$$

We deduce from Lemma 7 that

$$
\bar{y}_{t}(0, t) \underset{t \rightarrow \infty}{\longrightarrow} 0 \text {. }
$$

Consider now any $\bar{U}_{2} \in \omega\left(\bar{U}_{1}\right)$ and a sequence $t_{n} \rightarrow+\infty$ such that $\bar{S}\left(t_{n}\right) \bar{U}_{1} \rightarrow \bar{U}_{2}$ when $n \rightarrow+\infty$, and define $\bar{W}(t)=\left(\bar{y}_{2}(., t), \bar{z}_{2}(., t), \bar{\eta}_{2}(t), \bar{\xi}_{2}(t)\right)=\bar{S}(t) \bar{U}_{2}$. Fix any $t \geq 0$; since $\bar{S}(t)$ is a continuous operator on $H$ for all $t \geq 0$, we have

$$
\bar{V}\left(t+t_{n}\right)=\bar{S}(t)\left(\bar{V}\left(t_{n}\right)\right)=\bar{S}(t)\left(\bar{S}\left(t_{n}\right) \bar{U}_{1}\right) \underset{n \rightarrow \infty}{\longrightarrow} \bar{S}(t) \bar{U}_{2}=\bar{W}(t)
$$

In particular, looking at the third components,

$$
\bar{y}_{t}\left(0, t+t_{n}\right) \underset{n \rightarrow \infty}{\longrightarrow} \bar{\eta}_{2}(t)
$$


Comparison with (4.26) yields $\bar{\eta}_{2}(t)=0$. Thus $\bar{\eta}_{2} \equiv 0$ and by Lemma $6, \bar{W} \equiv 0$.

To conclude, let us observe that, in the one hand, $\left\|\bar{V}\left(t+t_{n}\right)\right\|^{2}$ is constant equal to $\|\bar{V}(0)\|^{2}=\left\|\bar{U}_{1}\right\|^{2}$ and, in the other hand, is converging to $\|\bar{W}(t)\|^{2}$ which is zero. So $\bar{U}_{1}=0$ and proof of Theorem 4 is complete.

Remark: the semigroup $(S(t))_{t>0}$ being possibly non contractive, we cannot use any density argument to deal with convergence, weak or strong, of weak solutions and the method used in Lemma 4 requires too much regularity for the weak solutions. The asymptotic behaviour of weak solutions is an open question.

\section{REFERENCES}

[1] B. D'Andréa-Novel, F. Boustany, F. Conrad and B. Rao, Feedback stabilization of a hybrid PDE-ODE system. Math. Control Signals Systems (1994) 1-22.

[2] H. Brézis, Opérateurs maximaux monotones et semi-groupes de contractions dans les espaces de Hilbert. North Holland (1973).

[3] C.M. Dafermos and M. Slemrod, Asymptotic behaviour of nonlinear contraction semi-groups. J. Funct. Anal. 13 (1973) 97-106.

[4] A. Mifdal, Stabilisation uniforme d'un système hybride, C.R. Acad. Sci. Paris, 324, Série I (1997) 37-42.

[5] A. Mifdal, Étude de la stabilité forte et uniforme d'un système hybride. Application à un modèle de pont roulant, Thèse de l'Université de Nancy I (1997).

[6] B. Rao, Decay estimates of solutions for a hybrid system of flexible structures. Eur. J. Appl. Math. (1993) 303-319.

[7] D.L. Russell, Decay rates for weakly damped systems in Hilbert space obtained with control-theoretic methods. J. Diff. Eq. 19 (1975) 344-370. 